TITLE:

\title{
Step-by-step unbalanced force iteration method for cable-strut structure with irregular shape
}

AUTHOR(S):

Ma, Qing; Ohsaki, Makoto; Chen, Zhihua; Yan, Xiangyu

\section{CITATION:}

Ma, Qing ...[et al]. Step-by-step unbalanced force iteration method for cable-strut structure with irregular shape. Engineering Structures 2018, 177: 331-344

\section{ISSUE DATE:}

2018-12-15

URL:

http://hdl.handle.net/2433/242991

\section{RIGHT:}

(c) 2018. This manuscript version is made avail able under the CC-BY-NC-ND 4.0 license

http://creativecommons.org/licenses/by-nc-nd/4.0.; The full-text file will be made open to the public on 15 December 2020 in accordance with publisher's 'Terms and Conditions for Self-Archiving'.; この論文は出版社版でありません。引 用の際には出版社版をご確認ご利用ください。; This is not the published version. Please cite only the published version. 


\title{
Step-by-Step Unbalanced Force Iteration Method for Cable-Strut Structure with Irregular Shape
}

\author{
Qing Ma ${ }^{\mathrm{a}, \mathrm{b}}$, Makoto Ohsaki ${ }^{\mathrm{b}}$, Zhihua Chen ${ }^{\mathrm{a},{ }^{*}}$, Xiangyu Yan ${ }^{\mathrm{a}, \mathrm{c}}$ \\ ${ }^{a}$ Department of Civil Engineering, Tianjin University, Tianjin, China \\ ${ }^{\mathrm{b}}$ Department of Architecture and Architectural Engineering, Kyoto University, Kyoto, Japan \\ ${ }^{c}$ Architectural Design and Research Institute, Tianjin University, Tianjin, China
}

\begin{abstract}
In the design process of a cable-strut structure, the desired shape is first defined and the prestress can be obtained if the geometry is feasible; otherwise, the geometry must be modified. Thus, the initial step for prestress calculation is to estimate the feasibility of the geometry. In this paper, a method called Unbalanced Force Iteration (UFI) is proposed to remove the unbalanced forces using the equilibrium and stiffness equations. Feasibility of the geometry can be judged by the convergence property of UFI. Self-stress modes can be directly obtained easily through UFI method, if initial geometry is feasible. For structures with infeasible initial geometry, the Step-by-Step UFI, which combines finite element analysis and UFI, is proposed to gradually move the nodes to feasible locations. Three examples of structures with feasible geometry and three examples of cable domes with irregular and infeasible initial geometry are presented to verify the ability of UFI and Step-by-Step UFI for designing new irregular and asymmetric cable-strut structures.
\end{abstract}

Keywords: cable-strut structure; tensegrity; prestress design; form finding

\section{Introduction}

Cable-strut structure is a lightweight roof structure covering a large space in the field of civil and architectural engineering. It consists of cables and struts, and are stiffened by introducing pretensioning forces in a self-equilibrium state. Therefore, the mechanical properties of a cablestrut structure is similar to those of a tensegrity structure ${ }^{[1,2]}$. Over the past 60 years, numerous amount of studies have been conducted for analysis and design of tensegrity structures ${ }^{[3,4]}$. 
However, a cable-strut structure does not obey the rigorous definition of a tensegrity structure, because a tensegrity structure should be self-standing without support, which is not acceptable for a roof structure.

A long-span cable-strut structure called cable dome was first applied by Geiger in the two Gymnasiums of Seoul Olympics ${ }^{[5]}$. The structural configuration was then called 'Geiger form'. Levy adopted another form of cable dome consisting of triangle units in the main Gymnasium of Atlanta Olympics (Georgia Dome) ${ }^{[6]}$, which was the largest cable dome up to now. The advantage of lightweight property makes it a popular structural system in the design of large span roofs. In the recent three decades more than ten large cable domes have been constructed, including famous works such as the Suncoast Dome, Redbird Arena, Taoyuan County Arena, La Plata Stadium, The Crown Coliseum, etc. ${ }^{[7-12]}$, and many new forms have been proposed and studied $^{[13-18]}$. However, the majority of existing cable dome projects are in symmetric circular form.

Determination of prestress is the key step in design of cable-strut structures ${ }^{[7]}$, because the stability and stiffness against external loads depend on prestress distribution. Furthermore, feasible prestress, or feasible self-stress mode, is defined as a set of member forces satisfying the self-equilibrium conditions and the structural requirement such that cables are in tension and struts are in compression ${ }^{[19]}$. If feasible prestress is able to be obtained, we regard that the geometry is feasible. The process for determination of prestress with given geometry and topology is known as prestress design, while the process of obtaining feasible geometry with topological and structural demands is referred to as form-finding ${ }^{[20]}$.

Various methods have been presented for finding feasible prestresses or self-stress modes, e.g., by Zhang et al. ${ }^{[21]}$, Pellegrino ${ }^{[19]}$, Yuan et al. ${ }^{[14]}$, Wang et al. ${ }^{[22]}$, Tran et al. ${ }^{[23]}$, Guo and $\mathrm{Jiang}^{[24]}$, Ye et $a l .{ }^{[25]}$. Number of self-stress modes can be easily obtained from the rank of equilibrium matrix ${ }^{[21]}$, which can be computed by Singular Value Decomposition (SVD). If there is only one self-stress mode, feasibility of the geometry can easily checked. If the initial geometry is not feasible, the form-finding process is needed. Many methods have also been 
proposed for the form-finding problem. Among them, Dynamic Relaxation Method (DRM) ${ }^{[25]}$ and Force Density Method (FDM ${ }^{[26]}$ are very efficient methods to find the geometry when the unstressed member length or force density is known. For form-finding problems with geometrical and structural constraints, many non-linear or numerical methods have been presented based on FDM/DRM ${ }^{[27-31]}$ or Finite Element Method (FEM) ${ }^{[23,32-34]}$.

The problems encountered in the practical design process of cable domes are (1) when asymmetric or irregular configuration is to be designed, a more efficient and direct way for estimation of feasibility is needed; (2) a simple form-finding method is needed for structural designers to find the feasible geometry closest to the initial one.

In this paper, a method called Unbalanced Force Iteration (UFI) is first proposed. The geometrical feasibility can be estimated by the convergence property of UFI. Self-stress modes and the statical indeterminacy, which is equal to the number of independent self-stress modes, can be obtained in a simple manner, if geometry is feasible. The Step-by-Step UFI, which combines UFI and FEA, is presented for the from-finding problem. The efficiency of the proposed methods is demonstrated in numerical examples of cable-strut structures with various symmetric and asymmetric shapes.

\section{Basic Formulations}

Basic formulations of equilibrium equations and stiffness matrices are presented for prestress design and form-finding analysis of cable-strut structures.

\subsection{Equilibrium equation}

Let $m$ and $n$ denote the numbers of elements and nodes, respectively. Connectivity of the structure can be defined by the connectivity matrix $C \in R^{n \times m}$. If the $k$ th element starts at node $i$ and ends at node $j$, the $(k, e)$ component $C_{k, e}$ of matrix $C$ is defined as:

$$
C_{k, e}= \begin{cases}+1 & (e=i) \\ -1 & (e=j) \\ 0 & \text { (others) }\end{cases}
$$


Let $\boldsymbol{x}, \boldsymbol{y}$ and $\boldsymbol{z}\left(\in R^{n \times 1}\right)$ denote the coordinate vectors of nodes in $x$-, $y$ - and $z$-directions, respectively. The vectors of coordinate differences in $x$-, $y$ - and $z$-directions of the two end nodes of members are denoted by $\boldsymbol{u}=\left(u_{1}, \ldots, u_{m}\right)^{\mathrm{T}}, \quad \boldsymbol{v}=\left(v_{1}, \ldots, v_{m}\right)^{\mathrm{T}}$ and $\boldsymbol{w}=\left(w_{1}, \ldots, w_{m}\right)^{\mathrm{T}}$, respectively, which are calculated by

$$
\left\{\begin{array}{l}
u=C x \\
v=C y \\
w=C z
\end{array}\right.
$$

The coordinate difference matrices $\boldsymbol{U}, \boldsymbol{V}$ and $\boldsymbol{W}\left(\in R^{m \times m}\right)$ are defined as

$$
\left\{\begin{array}{l}
\boldsymbol{U}=\operatorname{diag}(\boldsymbol{u}) \\
\boldsymbol{V}=\operatorname{diag}(\boldsymbol{v}) \\
\boldsymbol{W}=\operatorname{diag}(\boldsymbol{w})
\end{array}\right.
$$

Let $\boldsymbol{l}=\left(l_{1}, \ldots, l_{m}\right)^{\mathrm{T}}$ denote the vector consisting of length of each element including cables and struts. The $k$ th component $l_{k}$ is computed as

$$
l_{k}=\sqrt{u_{k}^{2}+v_{k}^{2}+w_{k}^{2}}
$$

The element length matrix $\boldsymbol{L}$ is defined as

$$
\boldsymbol{L}=\operatorname{diag}(\boldsymbol{l})
$$

Then the equilibrium matrix $\boldsymbol{D}^{0} \in R^{3 n \times m}$ including the support degree of freedom is obtained as

$$
\boldsymbol{D}^{0}=\left[\begin{array}{l}
\boldsymbol{C}^{\mathrm{T}} \boldsymbol{U} \boldsymbol{L}^{-1} \\
\boldsymbol{C}^{\mathrm{T}} \boldsymbol{V} \boldsymbol{L}^{-1} \\
\boldsymbol{C}^{\mathrm{T}} \boldsymbol{W} \boldsymbol{L}^{-1}
\end{array}\right]
$$

Let $f$ denote the number of degrees of freedom of the structure. Equilibrium matrix $\boldsymbol{D}^{0}$ is reduced to $\boldsymbol{D} \in R^{f \times m}$ after removing the rows corresponding to the fixed displacement components. Self-equilibrium conditions of the cable-strut structure is formulated with respect to the vector $\boldsymbol{t} \in R^{m \times 1}$ consisting of prestress of each element as 


$$
\mathrm{Dt}=\mathbf{0}
$$

\subsection{Linear stiffness matrix}

The linear elastic properties of pretensioned cable-strut structures are the same as those of trusses, when cables are appropriately tensioned. Therefore, we derive the linear stiffness matrix of trusses below. Let $\boldsymbol{k}_{e} \in R^{m \times 1}$ denote the element stiffness vector. The $k$ th component $k_{e}^{k}$ can be calculated by

$$
k_{e}^{k}=\frac{E A_{k}}{l_{k}}
$$

where $E$ is Young's modulus and $A_{k}$ is the cross-sectional area of the $k$ th element. A diagonal matrix $\boldsymbol{K}_{e} \in R^{m \times m}$ of element stiffness is defined as

$$
\boldsymbol{K}_{e}=\operatorname{diag}\left(\boldsymbol{k}_{e}\right)
$$

Let $\boldsymbol{d} \in R^{f \times 1}$ denote the nodal displacement vector of the structure subjected to the external load vector $\boldsymbol{F} \in R^{f \times 1}$. Elongation vector $\Delta \boldsymbol{l} \in R^{m \times 1}$ of members is calculated by

$$
\Delta \boldsymbol{l}=\boldsymbol{B d}
$$

where $\boldsymbol{B} \in R^{m \times 3 n}$ is the compatibility matrix, which is the transpose of the equilibrium matrix $\boldsymbol{D}$ as

$$
\boldsymbol{B}=\boldsymbol{D}^{\mathrm{T}}
$$

Then, the global linear stiffness matrix $\boldsymbol{K}_{E} \in R^{f \times f}$ after removing the support degree of freedom is obtained as

$$
\boldsymbol{K}_{E}=\boldsymbol{D} \boldsymbol{K}_{e} \boldsymbol{D}^{\mathrm{T}}
$$

The nodal displacement vector $\boldsymbol{d}$ under nodal loads $\boldsymbol{F}$ can be obtained from the following equation:

$$
\boldsymbol{K}_{E} \boldsymbol{d}=\boldsymbol{F}
$$

The resulting internal force can be calculated by 


$$
\boldsymbol{t}=\Delta \boldsymbol{l} \circ \boldsymbol{k}_{e}=\boldsymbol{K}_{e} \Delta \boldsymbol{l}=\boldsymbol{K}_{e} \boldsymbol{D}^{\mathrm{T}} \boldsymbol{d}
$$

with (o) denoting Hadamard product ${ }^{[35]}$, or element-wise product, of two vectors or matrices.

\section{Step-by-Step Unbalanced Force Iteration Method}

\subsection{Estimation of feasibility}

For the design of cable-strut structures, it is important that prestresses of cables and struts satisfy consistency in signs such that positive (tensile) force in cable and negative (compressive) force in strut. In this section, we propose a simple iterative approach to obtaining feasible presetresses of elements satisfying consistency in signs.

The prestress vector applied to the elements at the initial step of iteration is denoted by $\boldsymbol{t}_{0}$, which is randomly generated, or defined based on some intuition. If $\boldsymbol{t}_{0}$ does not satisfy the self-equilibrium conditions as Eq. (7), there will be unbalanced forces at nodes. The target of the iterative method called Unbalanced Force Iteration (UFI) is to eliminate the unbalanced force and to satisfy consistency in signs of forces of cables and struts.

The vector of unbalanced force $\boldsymbol{P}_{0} \in R^{f \times 1}$ can be obtained by

$$
\boldsymbol{P}_{0}=\boldsymbol{D} \boldsymbol{t}_{0}
$$

If nodal unbalanced force is properly re-distributed to each element, the contribution of elements to unbalanced force will be compensated and equilibrium state will be satisfied. The re-distribution process is conducted by applying unbalanced force $\boldsymbol{F}=-\boldsymbol{P}_{0}$ at nodes in opposite direction assuming all elements as truss elements. Using Eq. (13), the displacement vector $\boldsymbol{d}_{0}$ is obtained from

$$
-\boldsymbol{P}_{0}=\boldsymbol{K}_{E} \boldsymbol{d}_{0}
$$

which is rewritten as

$$
-\boldsymbol{P}_{0}=\boldsymbol{K}_{\mathrm{E}} \boldsymbol{d}_{0}=\boldsymbol{D} \boldsymbol{K}_{\mathrm{e}} \boldsymbol{D}^{\mathrm{T}} \boldsymbol{d}_{0}=\boldsymbol{D} \Delta \boldsymbol{t}_{0}
$$


where $\Delta t_{0}$ is the prestress force due to the unbalanced force. From Eqs. (15) and (17), we obtain

$$
D\left(t_{0}+\Delta t_{0}\right)=\mathbf{0}
$$

Thus, prestress is modified as follows to obtain a self-equilibrium prestress mode $\boldsymbol{t}_{1}$ :

$$
\boldsymbol{t}_{1}=\boldsymbol{t}_{0}+\Delta \boldsymbol{t}_{0}, \quad \boldsymbol{D} \boldsymbol{t}_{1}=\mathbf{0}
$$

The following results are obtained from this procedure:

1. If there exists a self-stress mode, it can be obtained by solving a stiffness equation only once. Therefore, we do not have to carry out SVD that demands more computational cost. As will be explained in Sec. 3.2, SVD is not needed also for calculating the number of independent self-stress modes.

2. Only linear stiffness matrix is to be used in this process, although the displacements of a prestressed structure is obtained using the tangent stiffness matrix that is the sum of linear and geometrical stiffness matrices, and only the geometrical stiffness matrix is usually used for form-finding analysis.

Let $\boldsymbol{T}^{c}=\left(T_{1}^{c}, \ldots, T_{m^{c}}^{c}\right)^{\mathrm{T}}$ and $\boldsymbol{T}^{s}=\left(T_{1}^{s}, \ldots, T_{m^{s}}^{s}\right)^{\mathrm{T}}$ denote the vectors consisting of forces of cables and struts, respectively, where $m_{c}$ and $m_{s}$ are the numbers of cables and struts. The modified prestress may not comply with the requirement that cables are tensioned and struts are compressed as follow:

$$
\begin{cases}T_{i}^{c}>0, & \left(i=1, \ldots, m_{c}\right) \\ T_{i}^{s}<0, & \left(i=1, \ldots, m_{s}\right)\end{cases}
$$

If Eq. (20) is not satisfied, the prestress $t_{1}$ obtained by Eq. (19) should be revised by a set of prestesses as follows:

$$
\left\{\begin{array}{l}
T_{i}^{c}=\left|T_{i}^{c}\right|, \quad\left(i=1, \ldots, m_{c}\right) \\
T_{i}^{s}=-\left|T_{i}^{s}\right|, \quad\left(i=1, \ldots, m_{s}\right)
\end{array}\right.
$$


Then, the process of Eqs. (15)-(21) is carried out again to update the self-stress mode.

The detail of process of UFI is as follows:

Step 1: Set the iteration counter $k=0$, and randomly assign the initial prestress vector $\boldsymbol{t}_{0}$.

Step 2: Obtain the unbalanced force $\boldsymbol{P}_{k}$ from $\boldsymbol{P}_{k}=\boldsymbol{D t}_{k}$, compute the nodal displacement vector $\boldsymbol{d}_{k}$ by $-\boldsymbol{P}_{k}=\boldsymbol{K}_{E} \boldsymbol{d}_{k}$, and find the self-equilibrium force vector $\boldsymbol{t}_{k+1}$ from $\Delta \boldsymbol{t}_{k}=\boldsymbol{K}_{\mathrm{e}} \boldsymbol{D}^{\mathrm{T}} \boldsymbol{d}_{k}$ and $\boldsymbol{t}_{k+1}=\boldsymbol{t}_{k}+\Delta \boldsymbol{t}_{k}$.

Step 3: Terminate the process if $\boldsymbol{t}_{k+1}$ is feasible; otherwise, update the signs of forces as Eq. (21), increase the counter $k \leftarrow k+1$, and go to Step 2 .

The flowchart of UFI is shown in Fig. 1. If the initial geometry is feasible, the unbalanced force $\boldsymbol{P}$ will converge to zero; otherwise, the iteration will not converge. Therefore, through the UFI process, the feasibility of initial geometry can be judged.

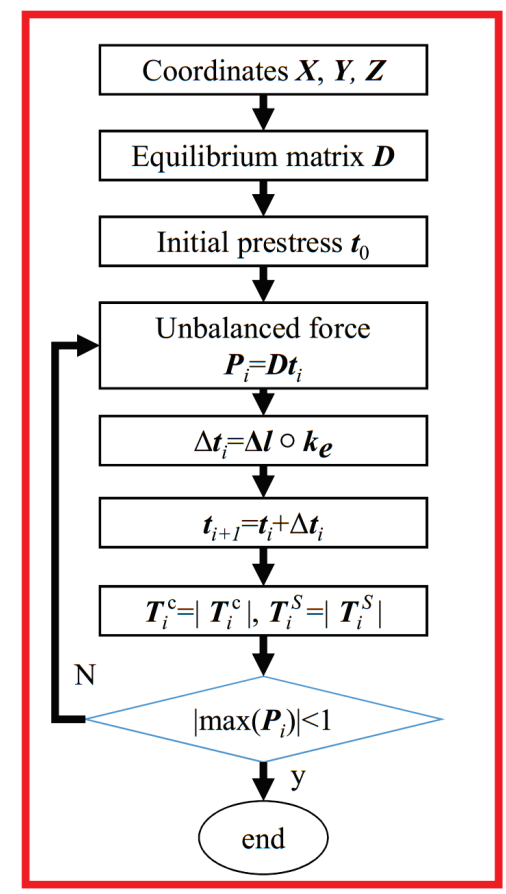

Fig.1 Flowchart of UFI 


\subsection{Obtaining self-stress modes}

If the cable-strut structure has a single self-stress mode, the unique self-stress mode can be directly obtained by single step of the UFI. However, thbtained mode of a structure with multiple self-stress modes can be assigned as an arbitrary linear combination of self-stress modes. If the UFI is converged, the following process can be utilized to obtain all independent self-stress modes and the number of statical indeterminacy, which is equal to the number of independent self-stress modes:

Step 1: Set the iteration counter $k=0$, and randomly assign the prestress $\boldsymbol{t}_{0}$. Compute $\Delta \boldsymbol{t}_{0}$ from Eqs. (15)-(19), and find the self-stress mode $\boldsymbol{s}_{1}^{f}=\boldsymbol{t}_{0}+\Delta \boldsymbol{t}_{0}$.

Step 2: Increase the iteration counter as $k \leftarrow k+1$, and randomly assign $\boldsymbol{t}_{k}$. Let $\boldsymbol{s}_{k+1}^{f}=\boldsymbol{t}_{k}$, and successively update $\boldsymbol{s}_{k+1}^{f}$ by

$$
\boldsymbol{s}_{k+1}^{f}=\boldsymbol{s}_{k+1}^{f}-\frac{\boldsymbol{s}_{i}^{f} \cdot \boldsymbol{s}_{k+1}^{f}}{\boldsymbol{s}_{i}^{f} \cdot \boldsymbol{s}_{i}^{f}} \boldsymbol{s}_{i}^{f}
$$

for $i=0, \ldots, k$ to find the self-stress mode that is orthogonal to the previously found selfstress modes.

Step 3: If $\left|\boldsymbol{s}_{k+1}^{f}\right|=0$, we obtain the number of self-stress modes as $\eta=k$, and the independent self-stress modes $\boldsymbol{s}_{k}^{f} \quad(k=1, \ldots, \eta)$. Otherwise, go to Step 2.

The algorithm for finding the self-stress modes is summarized in the flowchart in Fig. 2. This way, the number of self-stress modes can be obtained without resort to computationally expensive SVD. Note that this process can be incorporated into the iterative process of finding the feasible self-stress mode in Sec. 3.1. However, in this process, we do not have to consider the compatibility of signs of prestresses. 


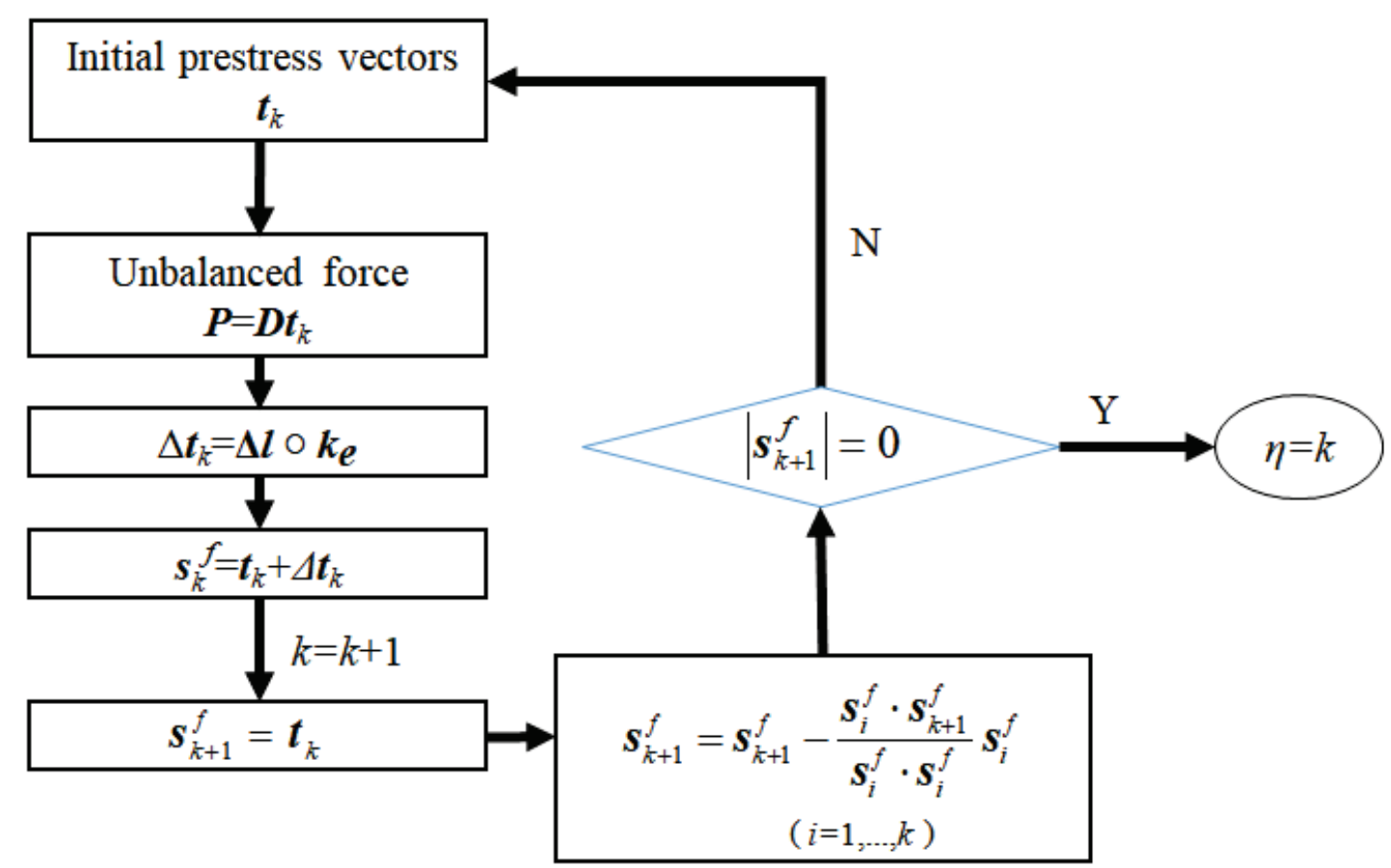

Fig.2 Flowchart for obtaining self-stress modes

\subsection{Geometry modification}

If the UFI cannot converge to reduce the unbalanced force to zero, i.e., the initial geometry is not feasible, modification of geometry is to be conducted through nonlinear FE analysis. Before the FE analysis, prestress must be revised in order to comply with the requirement of Eq. (20). However, direct use of Eq. (21) may cause the unbalanced force to be too large for nonlinear FE analysis to converge. Instead of Eq. (21), incompatible prestresses are adjusted to a positive constant $N_{1}$ for cable and a negative constant $N_{2}$ for strut, respectively, as

$$
\begin{cases}T_{i}^{c}=N_{1} & \text { for } T_{i}^{c}<0 \\ T_{i}^{s}=N_{2} & \text { for } T_{i}^{s}>0\end{cases}
$$

Let $\boldsymbol{t}^{*}$ denote the force vector after being updated by Eq. (23). Then, the unbalanced force vector $\boldsymbol{P}^{*}$ is calculated by

$$
\boldsymbol{P}^{*}=\boldsymbol{D} \boldsymbol{t}^{*}
$$


The increment of nodal coordinate vector $\boldsymbol{X}$, which is the displacement vector to cancel the unbalanced load, is denoted by $\boldsymbol{d}$. By differentiating the equilibrium equation with respect to $\boldsymbol{X}$, the following equation is obtained to calculate $\boldsymbol{d}$ :

$$
\boldsymbol{P}^{*}+\frac{\partial(\boldsymbol{D} \boldsymbol{t})}{\partial \boldsymbol{X}} \boldsymbol{d}=\mathbf{0} \quad \Rightarrow \quad \boldsymbol{K} \boldsymbol{d}=-\boldsymbol{P}^{*}
$$

where $\boldsymbol{K}$ is the global tangent stiffness matrix, which is defined as the sum of linear stiffness matrix expressed in Eq. (12) and the geometrical stiffness matrix due to prestress. The global tangent stiffness matrix in nonlinear analysis process is not constant, and it is updated at every step of solving Eq. (25).

Let $\boldsymbol{x}^{i}, \boldsymbol{y}^{i}$ and $\boldsymbol{z}^{i}$ denote the vectors consisting of $x$-, $y$ - and $z$-coordinates, respectively, of nodes at the $i$ th step of iteration. The increments of $x$-, $y$ - and $z$-coordinate vectors at the $i$ th step are denoted by $\boldsymbol{d}_{x}^{i}, \boldsymbol{d}_{y}^{i}$ and $\boldsymbol{d}_{z}^{i}$, respectively, which are obtained from $\boldsymbol{d}$. Note that the coordinates of some selected nodes are to be modified to prevent drastic change of selfequilibrium shape; therefore, most of the components of $\boldsymbol{d}_{x}^{i}, \boldsymbol{d}_{y}^{i}$ and $\boldsymbol{d}_{z}^{i}$ are zero. Thus, the nodal coordinates are updated as

$$
\left\{\begin{array}{l}
\boldsymbol{x}^{i+1}=\boldsymbol{x}^{i}+d_{x}^{i} \\
\boldsymbol{y}^{i+1}=\boldsymbol{y}^{i}+d_{y}^{i} \\
\boldsymbol{z}^{i+1}=\boldsymbol{z}^{i}+d_{z}^{i}
\end{array}\right.
$$

The geometry update process is summarized as follows:

Step1: Check feasibility of self-stress mode obtained by UFI. If it is not feasible, modify the forces using Eq. (23).

Step2: Calculate the unbalanced force by Eq. (24), and obtain displacement vector from Eq. (25).

Step3 Update the geometry using Eq. (26), and return to UFI.

For the given initial prestress and geometry, UFI is first processed. Then, compatibility of prestress will be checked and prestress will be revised using Eq. (23). The feasible prestress will be obtained until equilibrium and compatibility conditions of the signs of forces are both 
satisfied. The method for force and form finding is named Step-by-Step UFI. The flowchart of the whole method is shown in Fig. 3.

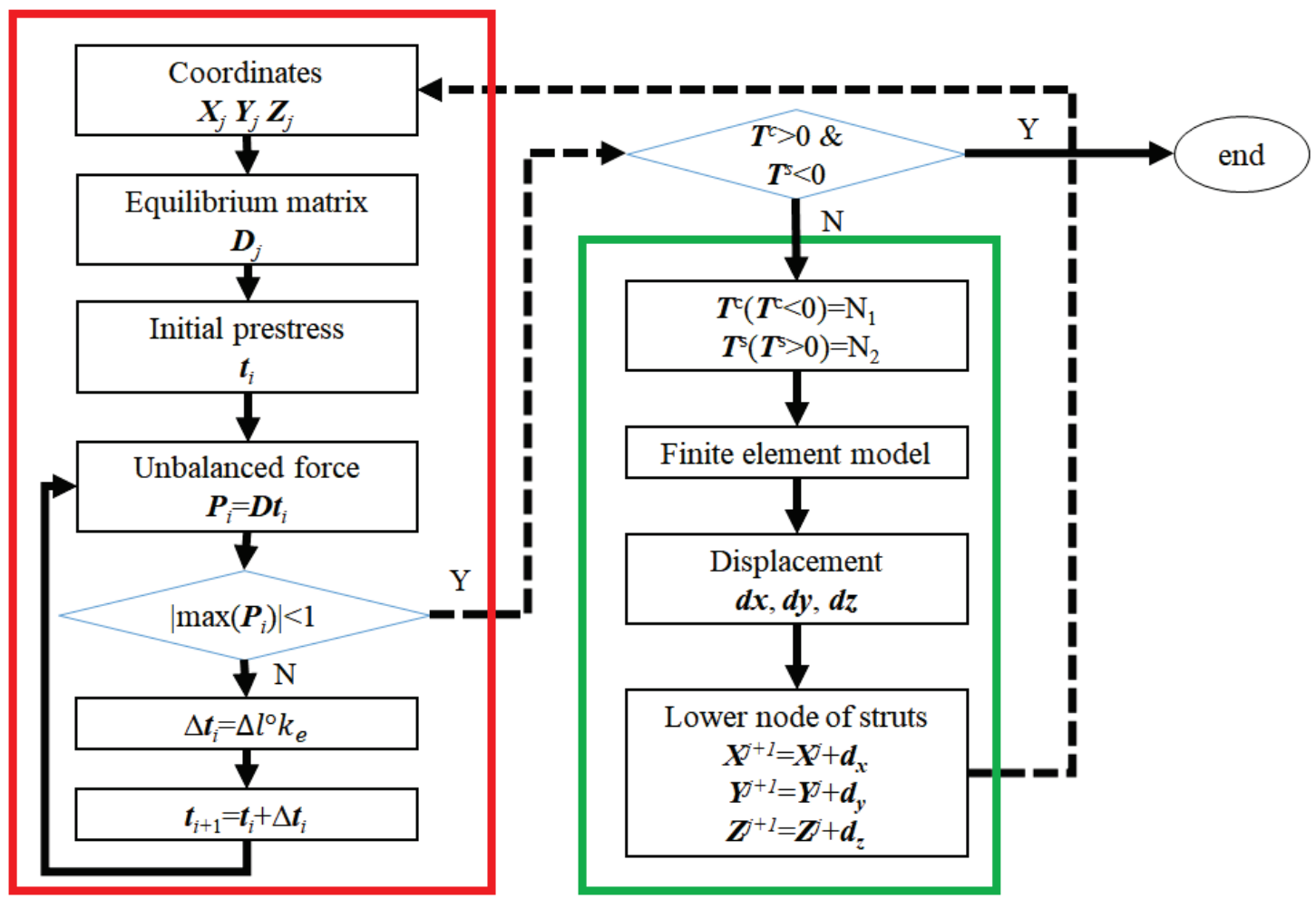

Fig. 3 Flowchart of Step-by-Step UFI; red box is UFI process and green box is geometry update process.

\section{Examples}

Numerical examples are presented for cable-strut structures with symmetric and feasible geometry (Examples I, II and III) and asymmetric and infeasible initial geometry (Examples IV, $\mathrm{V}$ and VI). To verify that the UFI method shown in the red box in Fig. 3 is able to judge feasibility of geometry, random values are first assigned for prestresses of cables and struts, and the results of UFI are compared with those in existing papers. The UFI process is done with Matlab Ver. $2016^{[36]}$ and FEA by Ansys Ver. $18^{[37]}$. Examples I and II are circular Geiger and Levy form cable domes, respectively. It is known that they both have single self-stress mode. Convergence tolerance of all six examples are set to $1.0 \mathrm{~N}$. 


\subsection{Cable domes with feasible geometry}

\section{Example I: Circular Geiger form cable dome}

Figure 4 shows the top view, perspective view and the element group numbers of a unit of a circular Geiger form cable dome with diameter $100 \mathrm{~m}$. By utilizing symmetry conditions, the whole structure is divided into 12 units with the same topology and geometry. There are three rings of hoops and 37 struts and 132 cables in total. The cables and struts in each unit are classified into 18 element groups as shown in Fig. 4(c), where groups 1, 2, 4, 5, 8 and 9 are ring cables, groups 15-18 are struts and others are cables.

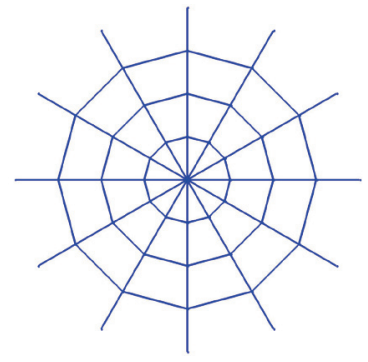

(a) top view

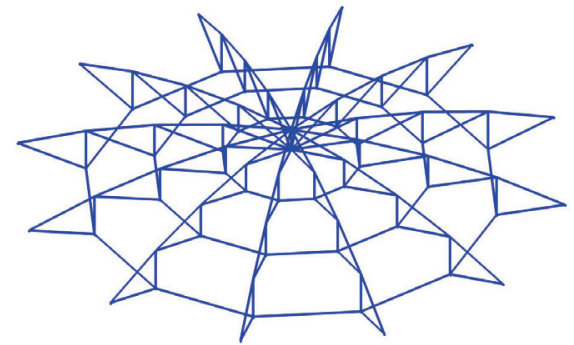

(b) perspective view

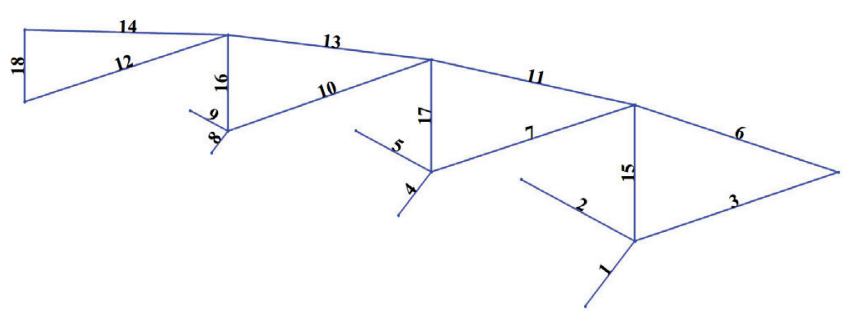

(c) element group numbers of a unit

Fig. 4 Circular Geiger form cable dome

The prestresses of elements designated in Fig. 4(c) are listed in Table 1. The results by UFI almost coincide with those obtained by SVD. The maximum unbalanced force calculated from the initial prestresses is $5.75 \times 10^{6} \mathrm{~N}$. The unbalanced force vanishes to $1.01 \times 10^{-5} \mathrm{~N}$, which is far below the convergence tolerance, with only one step of UFI without geometry modification. The results in Tables 1 verify that this cable dome is feasible, and has only one self-stress mode. 
Table 1 UFI results of circular Geiger form cable dome

\begin{tabular}{|c|c|c|c|c|c|c|c|c|c|}
\hline Ele. & Intial value & UFI (N) & SVD (N) & Error (N) & No. & Intial value & UFI (N) & SVD (N) & Error (N) \\
\hline 1 & 250000.0 & 250000.0 & 250000.0 & 0 & 10 & 67812.1 & 20799.3 & 20799.3 & $-1.16 \mathrm{e}-09$ \\
\hline 2 & -117797.0 & 250000.0 & 250000.0 & $-7.45 \mathrm{e}-09$ & 11 & -30159.5 & 66854.9 & 66854.9 & $-4.56 \mathrm{e}-08$ \\
\hline 3 & 66824.9 & 135956.4 & 135956.4 & $-1.49 \mathrm{e}-08$ & 12 & -480924.3 & 6332.8 & 6332.8 & $-3.09 \mathrm{e}-09$ \\
\hline 4 & -417912.0 & 99619.9 & 99619.9 & $-1.58 \mathrm{e}-08$ & 13 & -160483.5 & 45640.5 & 45640.5 & $-4.38 \mathrm{e}-08$ \\
\hline 5 & -439494.2 & 99619.9 & 99619.9 & $-1.49 \mathrm{e}-08$ & 14 & -332852.7 & 39161.8 & 39161.8 & $-4.42 \mathrm{e}-08$ \\
\hline 6 & 30344.9 & 123691.1 & 123691.1 & $-5.03 \mathrm{e}-08$ & 15 & 289959.4 & -41681.3 & -41681.3 & $6.05 \mathrm{e}-09$ \\
\hline 7 & 275064.3 & 54019.1 & 54019.1 & $-5.59 \mathrm{e}-09$ & 16 & -186010.4 & -6590.4 & -6590.4 & $9.26 \mathrm{e}-09$ \\
\hline 8 & 427632.0 & 38110.7 & 38110.7 & $2.79 \mathrm{e}-09$ & 17 & 28113.8 & -16090.6 & -16090.6 & $-1.16 \mathrm{e}-10$ \\
\hline 9 & -364654.5 & 38110.7 & 38110.7 & $1.86 \mathrm{e}-09$ & 18 & -329437.3 & -1885.1 & -1885.1 & $-7.71 \mathrm{e}-10$ \\
\hline
\end{tabular}

\section{Example II: Circular Levy form cable dome}

Consider the Levy dome as shown in Fig. 5 with diameter $80 \mathrm{~m}$. The detailed geometry is shown in Fig.5(c) with the 15 groups of elements, where groups 1, 6, 10 and 14 are struts, and others are cables. There are three rings of hoops in this example, and circumference of each hoop is divided into six parts.

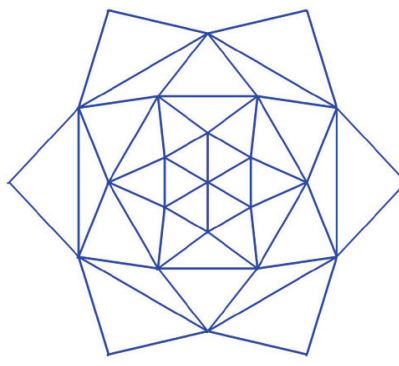

(a) top view
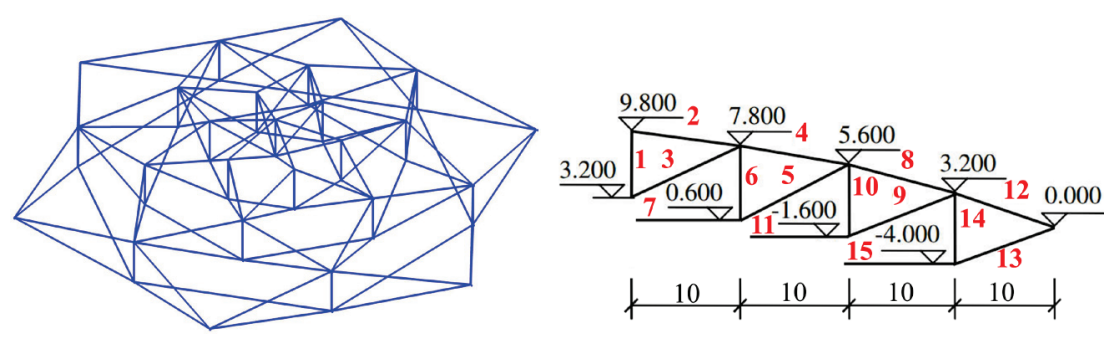

(b) perspective view

(c) geometry and member numbers

Fig. 5 Circular Levy form cable dome 
Table 2 UFI results of circular Levy form cable dome

\begin{tabular}{|c|c|c|c|c|c|c|c|c|c|}
\hline Ele. & Intial value & UFI (N) & $\begin{array}{c}\text { DSVD (N) } \\
{[14]}\end{array}$ & Error (N) & $\begin{array}{c}\text { Ele. } \\
\text { No. }\end{array}$ & Intial value & UFI (N) & $\begin{array}{c}\text { DSVD (N) } \\
{[14]}\end{array}$ & Error (N) \\
\hline 1 & -2500000.0 & -2500000.0 & -2500000.0 & 0 & 9 & -1526193.5 & 4695333.2 & 4695333.2 & $-1.38 \mathrm{e}-02$ \\
\hline 2 & 2935689.2 & 2124591.5 & 2124591.5 & $-5.83 \mathrm{e}-04$ & 10 & 1907147.5 & -2675618.4 & -2675618.4 & $5.49 \mathrm{e}-03$ \\
\hline 3 & -3148400.1 & 997035.4 & 997035.4 & $-3.37 \mathrm{e}-04$ & 11 & -435913.7 & 3333799.4 & 3333799.4 & $-2.39 \mathrm{e}-03$ \\
\hline 4 & 1747250.5 & 2569757.5 & 2569757.5 & $-3.41 \mathrm{e}-03$ & 12 & 2609956.1 & 47467213.6 & 47467213.6 & $-8.56 \mathrm{e}-02$ \\
\hline 5 & 2016686.5 & 1200479.4 & 1200479.4 & $-3.53 \mathrm{e}-03$ & 13 & -2022087.3 & 38226219.8 & 38226219.8 & $-7.55 \mathrm{e}-02$ \\
\hline 6 & 2343315.8 & -898310.2 & -898310.2 & $1.37 \mathrm{e}-03$ & 14 & -1501199.6 & -14619850.8 & -14619850.8 & $2.76 \mathrm{e}-02$ \\
\hline 7 & -2641204.3 & 1315217.4 & 1315217.4 & $-1.64 \mathrm{e}-03$ & 15 & -2252850.6 & 16962740.9 & 16962740.9 & $-2.55 \mathrm{e}-02$ \\
\hline 8 & -636952.2 & 9100297.5 & 9100297.5 & $-1.66 \mathrm{e}-02$ & & & & & \\
\hline
\end{tabular}

In the similar manner as the previous example of circular Geiger form, the prestresses of circular Levy form can be obtained with only one step of UFI. The final unbalanced force is $4.63 \times 10^{-5} \mathrm{~N}$, which is negligibly small. Table 2 shows the results of UFI and comparison between UFI and DSVD ${ }^{[14]}$. The UFI result is very close to DSVD result.

Table 2 verifies that the updated prestress always converges to the same value, because the geometry is feasible and the prestress mode is unique. Consequently, the unbalanced forces are directly eliminated, and the process of geometry update will not be called. Thus, it can be concluded from Examples I and II that for a single self-stress mode structure, the unique prestress mode can be obtained directly from an arbitrary initial value by only one step of UFI.

\section{Example III: Circular Kiewitt form cable dome}

The Kiewitt dome is designed by dividing the plane into six equal parts. There are two rings of hoops. The diameter of boundary is $60 \mathrm{~m}$, and the diameters of hoops are $20 \mathrm{~m}$ and $40 \mathrm{~m}$, respectively. The geometry is feasible and has four self-stress modes considering symmetry ${ }^{[20]}$. 
Figure 6(a) and (b) show the top and perspective views of the Kiewitt dome, respectively. The element group numbers of a unit are shown in Fig. 6(c), where the groups 15, 16, 17 and 18 are struts, and others are cables.

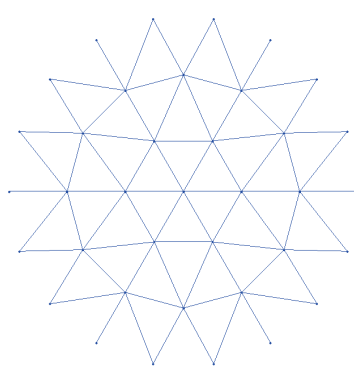

(a) top view

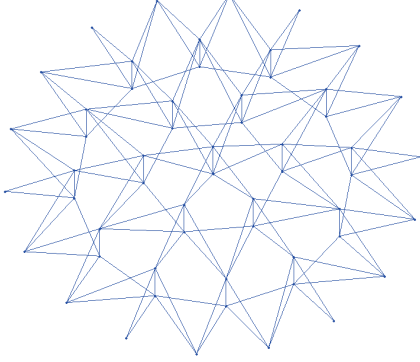

(b) perspective view

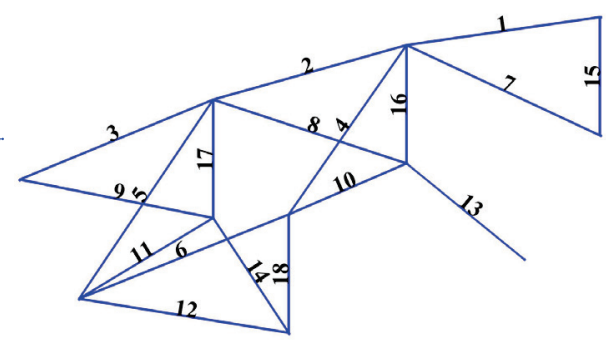

(c) element group numbers of a unit

Fig.6 Circular Kiewitt form cable dome

Table 3 UFI results of circular Kiewitt form cable dome

\begin{tabular}{|c|c|c|c|c|c|c|c|c|c|}
\hline Ele. & UFI(1) & UFI(2) & Ref. [23] & Ref. [14] & Ele. & UFI(1) & UFI(2) & Ref. [23] & Ref. [14] \\
\hline 1 & 435580 & 355826 & 3532423 & 369237 & 10 & 108038 & 69092 & 69262 & 65618 \\
\hline 2 & 506049 & 231530 & 228369 & 234963 & 11 & 185444 & 311697 & 257988 & 341831 \\
\hline 3 & 518827 & 126150 & 146112 & 290230 & 12 & 270848 & 270848 & 270848 & 275315 \\
\hline 4 & 0.03 & 152218 & 151554 & 164343 & 13 & 206427 & 209123 & 206962 & 221044 \\
\hline 5 & 85086 & 203690 & 183664 & 99060 & 14 & 875308 & 875308 & 875308 & 875308 \\
\hline 6 & 111827 & 238329 & 237778 & 251755 & 15 & -229101 & -187153 & -185363 & -186758 \\
\hline 7 & 67566 & 55195 & 54667 & 56529 & 16 & -127798 & -121209 & -120137 & -125512 \\
\hline 8 & 99384 & 150186 & 147550 & 168373 & 17 & -173870 & -185512 & -180560 & -198743 \\
\hline 9 & 242686 & 81494 & 150067 & 60432 & 18 & -164256 & -164256 & -164256 & -173178 \\
\hline
\end{tabular}




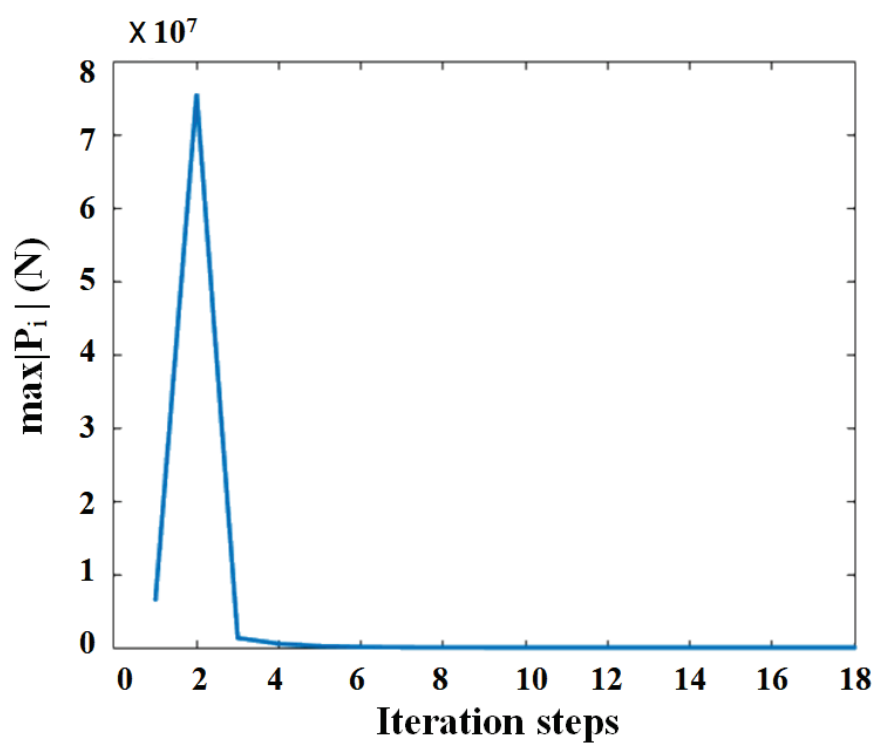

Fig. 7 Iteration history of UFI for Kiewitt form cable dome

Although the feasibility of this dome has been verified by Yuan ${ }^{[20]}$, UFI is first to be applied to verify its validity. Column UFI(1) in Table 3 is the result of randomly assigned initial values and column UFI(2) is the result obtained from the initial value such that the prestress of all cables are $250000 \mathrm{~N}$ and all struts are $-50000 \mathrm{~N}$. The history of unbalanced force with random initial value during UFI is shown in Fig. 6, while UFI(2) was obtained with only once step of UFI. As is shown in Fig. 7, UFI(1) converged at the 18th step with the final error $0.86 \mathrm{~N}$. Therefore, if the geometry is feasible, the UFI will converge from arbitrary random initial prestresses.

Pretresses in the two references are also listed in Table 3. For convenience of comparison, prestresses are normalized so that the value of element 14 is $875308 \mathrm{~N}$ for all three cases. As seen in Table 3, the three self-stress modes are different, since the Kiewitt form cable dome has multiple self-stress modes. The UFI can directly derive a group of feasible prestress for multiple self-stress mode structures. However, the result of UFI strongly depends on the initial values. Therefore, we generate all self-equilibrium modes using the procedure described in Sec. 3.2. 
Table 4 Self-stress modes of Kiewitt form cable dome obtained by UFI and DSVD

\begin{tabular}{|c|c|c|c|c|c|c|c|c|}
\hline & \multicolumn{4}{|c|}{ UFI } & \multicolumn{4}{|c|}{ DSVD } \\
\hline & $\boldsymbol{s}_{1}^{f}$ & $\boldsymbol{s}_{2}^{f}$ & $\boldsymbol{s}_{3}^{f}$ & $\boldsymbol{s}_{4}^{f}$ & $\boldsymbol{s}_{1}^{f}$ & $\boldsymbol{s}_{2}^{f}$ & $\boldsymbol{s}_{3}^{f}$ & $\boldsymbol{s}_{4}^{f}$ \\
\hline 1 & 1.00 & 1.00 & 1.00 & 1.00 & 1.00 & 1.00 & 1.00 & 1.00 \\
\hline 2 & 0.54 & 15.50 & 2.41 & -1.61 & -0.42 & 0.09 & 3.22 & 1.60 \\
\hline 3 & 2.73 & -5.86 & -1.45 & 25.10 & 6.05 & -0.86 & 1.29 & 6.68 \\
\hline 4 & 0.52 & -12.00 & -1.04 & 2.32 & 1.32 & 0.90 & -1.72 & -0.37 \\
\hline 5 & -1.33 & 12.43 & 2.74 & -18.56 & -4.04 & 1.17 & 1.17 & -3.83 \\
\hline 6 & 0.76 & -10.64 & -0.62 & 1.47 & 1.23 & 1.16 & -1.38 & -0.09 \\
\hline 7 & 0.16 & 0.16 & 0.16 & 0.16 & 0.16 & 0.16 & 0.16 & 0.16 \\
\hline 8 & 0.46 & -6.34 & -0.46 & 2.95 & 1.32 & 0.58 & -0.61 & 0.05 \\
\hline 9 & 3.30 & 3.84 & 1.61 & -12.80 & -5.10 & 1.03 & -0.60 & 6.77 \\
\hline 10 & 0.18 & 2.43 & 0.51 & -1.04 & -0.21 & 0.17 & 0.50 & 0.30 \\
\hline 11 & -1.48 & -5.24 & -0.43 & 8.47 & 4.42 & 0.57 & 0.66 & -4.59 \\
\hline 12 & 0.80 & -1.61 & 0.60 & -1.12 & 0.31 & 1.00 & 0.14 & 0.51 \\
\hline 13 & 0.61 & -2.99 & 0.14 & 1.49 & 0.94 & 0.70 & 0 & 0.37 \\
\hline 14 & 2.57 & -5.20 & 1.95 & -3.63 & 1.00 & 3.22 & 0.45 & 1.66 \\
\hline 15 & -0.53 & -0.53 & -0.53 & -0.53 & -0.53 & -0.53 & -0.53 & -0.53 \\
\hline 16 & -0.35 & 1.03 & -0.18 & -0.55 & -0.44 & -0.39 & -0.10 & -0.25 \\
\hline 17 & -0.32 & 1.41 & -0.31 & -0.13 & -0.59 & -0.63 & -0.14 & 0.13 \\
\hline 18 & -0.48 & 0.98 & -0.37 & 0.68 & -0.19 & -0.61 & -0.08 & -0.31 \\
\hline
\end{tabular}

The number of self-stress modes derived by the process in Fig. 2 is four, which is the same as the value obtained by DSVD ${ }^{[14]}$. The results of UFI and DSVD are listed in Table 4. It seems that the two results are different, because arbitrary linear combination of the self-stress modes is also a self-stress mode. To confirm that the results by UFI and SVD are the same, the reduced row echelon form of the matrices of self-stress modes are compared and listed in Table 5. The 
maximum error between UFI and DSVD is $4.69 \times 10^{-12} \mathrm{~N}$; therefore, self-stress modes obtained by UFI and DSVD are equivalent.

Table 5 Reduced row echelon form of self-stress modes of Kiewitt form cable dome obtained by UFI and DSVD

\begin{tabular}{|c|c|c|c|c|c|c|c|c|}
\hline & \multicolumn{4}{|c|}{ UFI } & \multicolumn{4}{|c|}{ DSVD } \\
\hline & $\boldsymbol{s}_{1}^{f}$ & $\boldsymbol{s}_{2}^{f}$ & $\boldsymbol{s}_{3}^{f}$ & $\boldsymbol{s}_{4}^{f}$ & $\boldsymbol{s}_{1}^{f}$ & $\boldsymbol{s}_{2}^{f}$ & $\boldsymbol{s}_{3}^{f}$ & $\boldsymbol{s}_{4}^{f}$ \\
\hline 1 & 1.00 & 0 & 0 & 0 & 1.00 & 0 & 0 & 0 \\
\hline 2 & 0 & 1.00 & 0 & 0 & 0 & 1.00 & 0 & 0 \\
\hline 3 & 0 & 0 & 1.00 & 0 & 0 & 0 & 1.00 & 0 \\
\hline 4 & 0.97 & -0.84 & 0 & 0 & 0.97 & -0.84 & 0 & 0 \\
\hline 5 & 0 & 0 & 0 & 1.00 & 0 & 0 & 0 & 1.00 \\
\hline 6 & 1.61 & -0.37 & -0.64 & -0.82 & 1.61 & -0.37 & -0.64 & -0.82 \\
\hline 7 & 0.16 & 0 & 0 & 0 & 0.16 & 0 & 0 & 0 \\
\hline 8 & 0 & -1.09 & 1.03 & 1.33 & 0 & -1.09 & 1.03 & 1.33 \\
\hline 9 & 18.98 & 18.05 & -27.08 & -36.49 & 18.98 & 18.05 & -27.08 & -36.49 \\
\hline 10 & 0.52 & 0.53 & -0.62 & -0.80 & 0.52 & 0.53 & -0.62 & -0.80 \\
\hline 11 & -12.17 & -13.04 & 19.07 & 25.82 & -12.17 & -13.04 & 19.07 & 25.82 \\
\hline 12 & 1.94 & 0.79 & -1.55 & -1.99 & 1.94 & 0.79 & -1.55 & -1.99 \\
\hline 13 & 0.57 & -0.39 & 0.25 & 0.32 & 0.57 & -0.39 & 0.25 & 0.32 \\
\hline 14 & 6.27 & 2.54 & -5.00 & -6.45 & 6.27 & 2.54 & -5.00 & -6.45 \\
\hline 15 & -0.53 & 0 & 0 & 0 & -0.53 & 0 & 0 & 0 \\
\hline 16 & -0.40 & 0.09 & 0 & 0 & -0.40 & 0.09 & 0 & 0 \\
\hline 17 & 0 & 0.75 & -0.86 & -1.23 & 0 & 0.75 & -0.86 & -1.23 \\
\hline 18 & -1.18 & -0.48 & 0.94 & 1.21 & -1.18 & -0.48 & 0.94 & 1.21 \\
\hline
\end{tabular}

\subsection{Cable domes with infeasible geometry}

It is observed from the above three examples that the UFI is applicable to structures with 
feasible initial geometry. The applicability of Step-by-Step UFI to infeasible initial geometry will be discussed in the following examples of three non-circular or asymmetric cable domes. The convergence criterion for Step-by-Step UFI is that maximum unbalanced force is within the tolerance value $1.0 \mathrm{~N}$.

\section{Example IV：Drop-shape Kiewitt form cable dome}

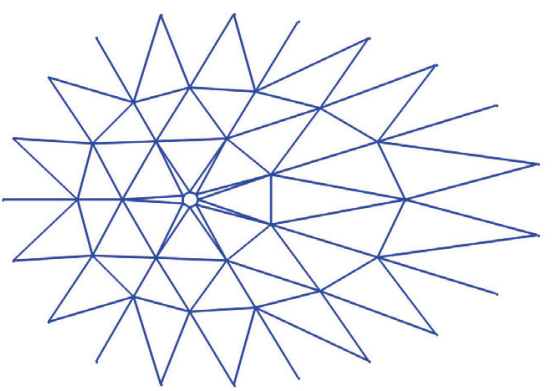

(a) top view

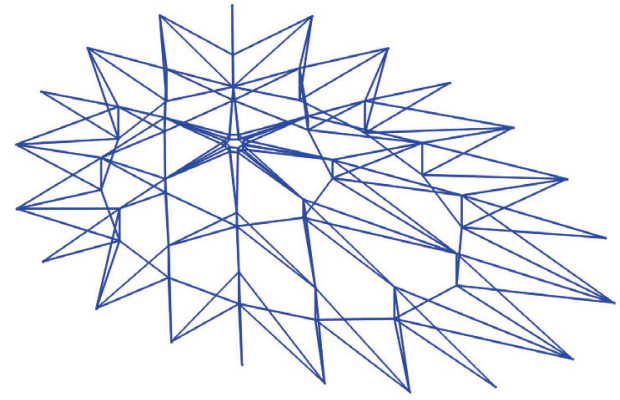

(b) perspective view

Fig. 8 Drop-shape Kiewitt form cable dome

Figure 8(a) and (b) show the top and perspective views, respectively, of a Kiewitt form cable dome with two rings of hoops and an inner ring based on a drop-shaped plane. The major axis of boundary is $89.5 \mathrm{~m}$ and the minor axis is $61.2 \mathrm{~m}$. There are 175 cables and 28 struts. On the boundary, there are 21 nodes that are all pin-supported. In the initial geometry, nodes on the same ring of hoop are all at the same height.

The history of UFI is shown in Fig. 9, which shows that the unbalanced force does not converge to zero and is still as large as $4.9 \times 10^{5} \mathrm{~N}$ after 100 steps of iteration. Thus, it can be judged that the initial geometry is not feasible, and we carry out Step-by-Step UFI. The $x$-, $y$ and $z$-coordinates of the nodes on hoops and the lower nodes of inner ring are considered as variables during Step-by-Step UFI. The locations of other nodes are fixed to guarantee the surface shape of the dome close to the original shape. 


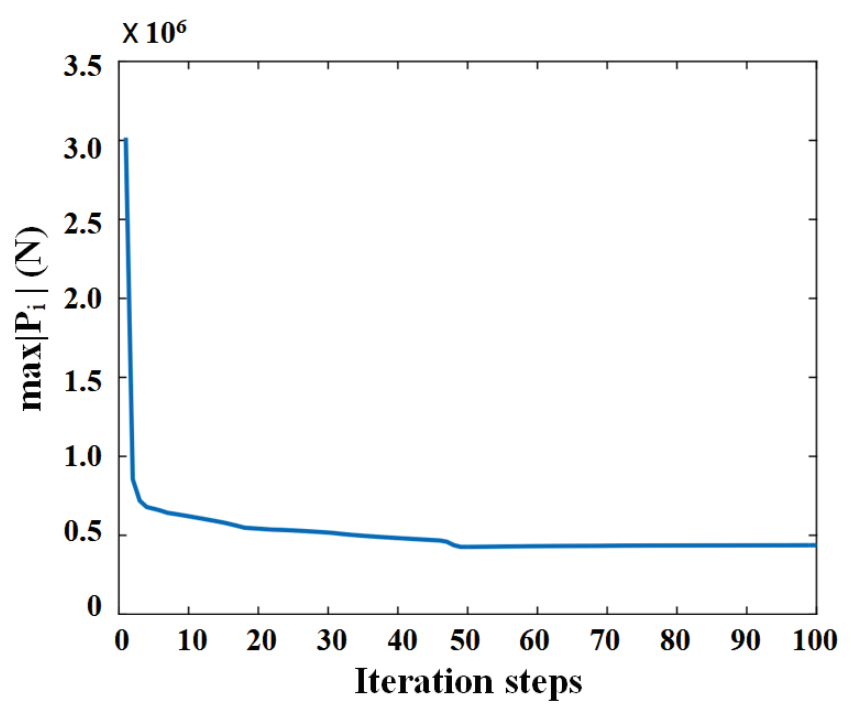

Fig.9 History of UFI of drop-shape Kiewitt form cable dome

The initial prestress values for Step-by-Step UFI are assigned as $\boldsymbol{T}_{c}^{0}=250000 \mathrm{~N}$ for cables and $\boldsymbol{T}_{s}^{0}=-50000 \mathrm{~N}$ for struts. The correction value defined as $N_{1}$ and $N_{2}$ in the green box of Fig. 3 are set as $N_{1}=5000 \mathrm{~N}$ and $N_{2}=-5000 \mathrm{~N}$, respectively. Step-by-Step UFI converged at the 45 th step with the unbalanced force $1.06 \times 10^{-6} \mathrm{~N}$. The history of iteration is shown in Fig. 10.

Average distance between initial and final nodal locations is $0.467 \mathrm{~m}$, and the maximum distance is $1.13 \mathrm{~m}$, which are small enough. It can be seen from Fig. 11 that the horizontal locations of all nodes are almost the same except the two nodes. In vertical direction, height differences for hoops are also not quite significant, and the largest difference exists at lower nodes of inner ring, for which the height varies between $1.02 \mathrm{~m}$ and $1.13 \mathrm{~m}$. However, that difference does not have much effect on the clearance of the roof. 


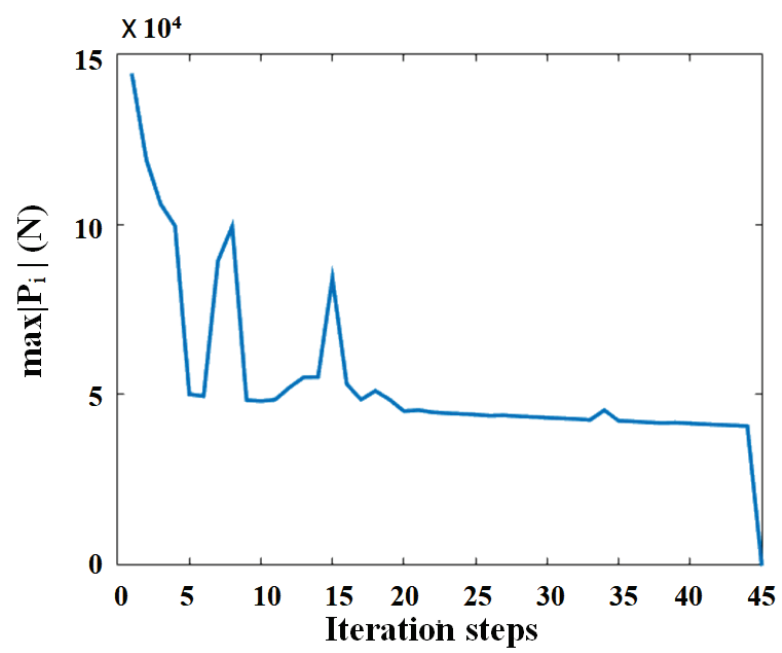

Fig. 10 History of Step-by-Step UFI of drop-shape Kiewitt form cable dome

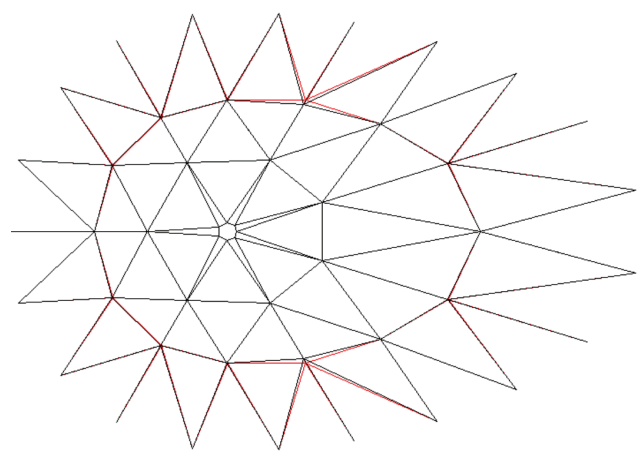

(a) top view

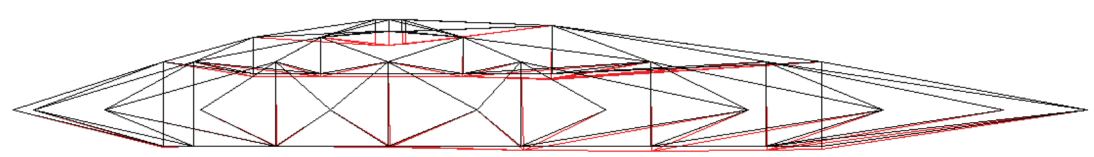

(b) vertical view

Fig.11 Comparison of initial and final geometries (red: initial; black: final)

\section{Example V: Ellipsoidal Levy form cable dome}

The ellipsoidal Levy form cable dome, as shown in Fig. 12, has a major axis of $102 \mathrm{~m}$ and minor axis of $83 \mathrm{~m}$. There are three rings of hoops and an inner ring; the total numbers of cables and struts are 272 and 92, respectively. Each hoop is divided into 16 parts. Nodes on the same hoop are all at the same height in the initial geometry. Locations of 64 nodes on the hoops and 
the lower inner ring are considered as variables. The nodes on boundary of the structure are not at the same height; the highest point is at ends of minor axis and the lowest at poles of major axis. The height difference is $5.45 \mathrm{~m}$. Figure 12(a) and (b) show the top and perspective views, respectively, of the ellipsoidal Levy form cable dome.

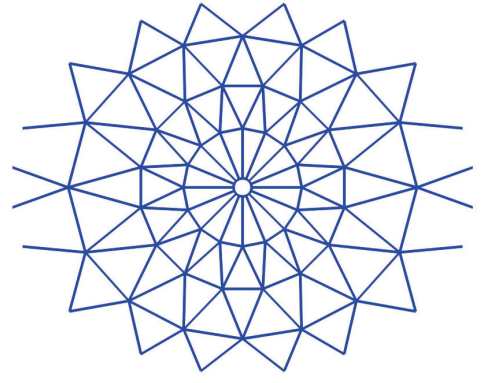

(a) top view

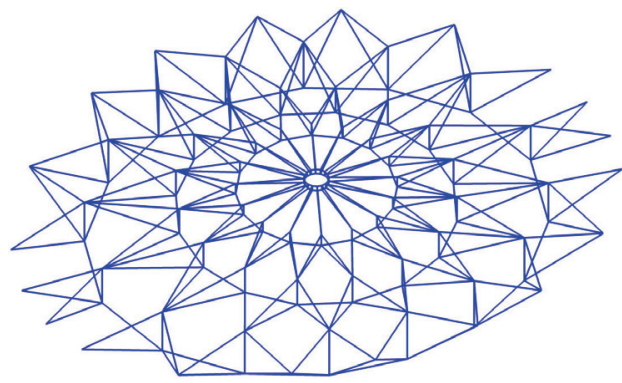

(b) perspective view

Fig.12 Ellipsoid Levy form cable dome

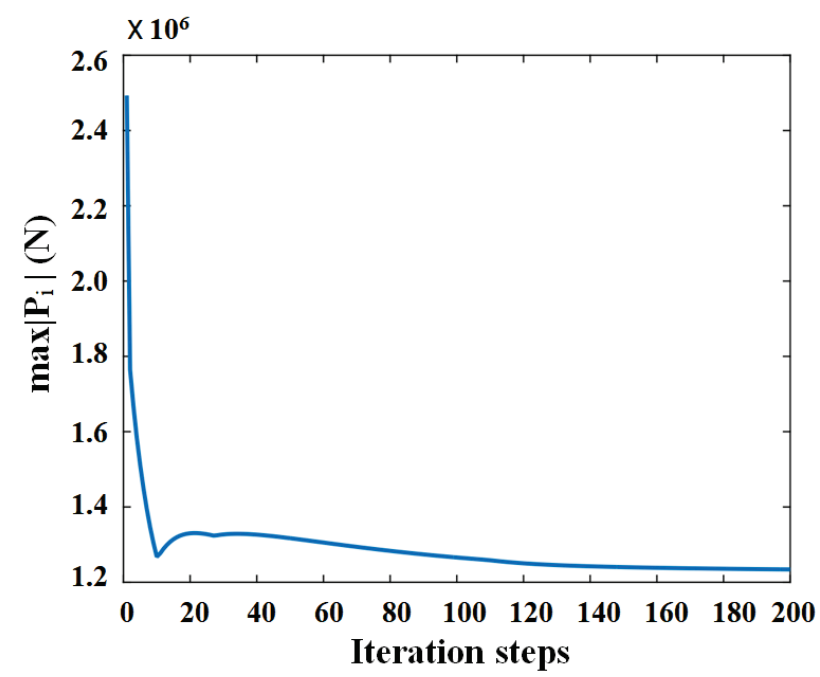

Fig.13 History of UFI of initial geometry of ellipsoidal Levy form cable dome

The initial prestress values of cables and struts are $250000 \mathrm{~N}$ and $-50000 \mathrm{~N}$, respectively.

The history of UFI result is shown in Fig. 13. Unbalanced force does not converge to zero after 200 steps of iteration; therefore the initial geometry is infeasible.

The values of $N_{1}$ and $N_{2}$ are set as $5000 \mathrm{~N}$ and $-5000 \mathrm{~N}$, respectively. Step-by-Step UFI 
converged at the 49th iteration with the final unbalanced force $0.98 \mathrm{~N}$. The initial and final geometries are compared in Fig. 14. The history of iteration is shown in Fig. 15. The horizontal coordinates of the two models are almost coincide with each other, while the hoops are no longer at the same height. The middle and the outmost hoops deformed into curved shapes similar to the boundary.

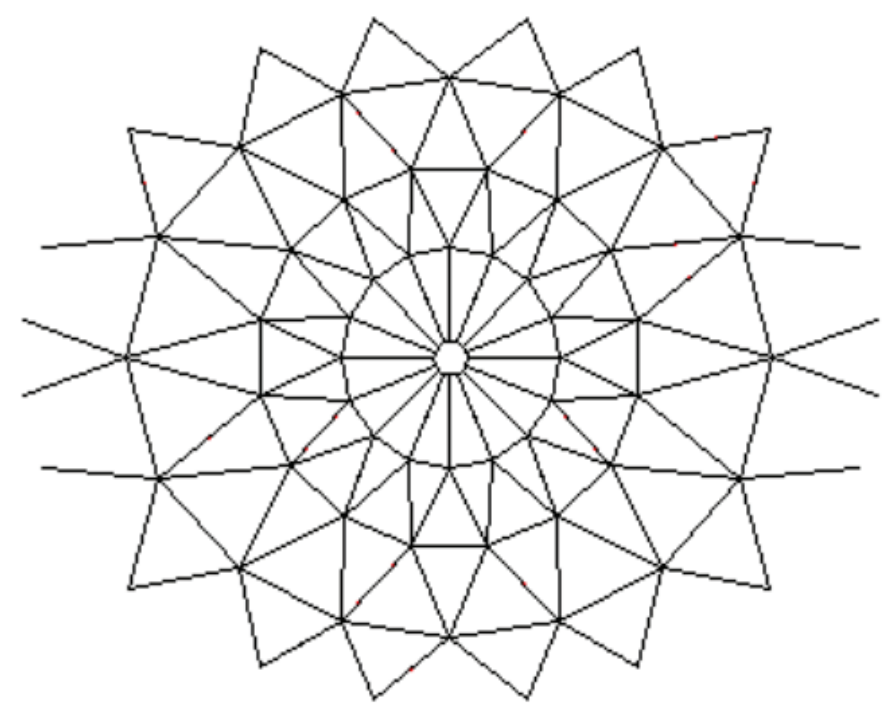

(a) top view

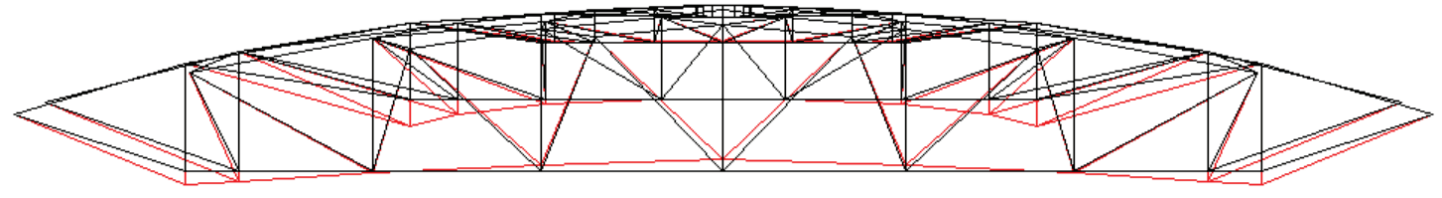

(b) vertical view

Fig.14 Comparison of initial and final geometries (red: initial; black: final) 


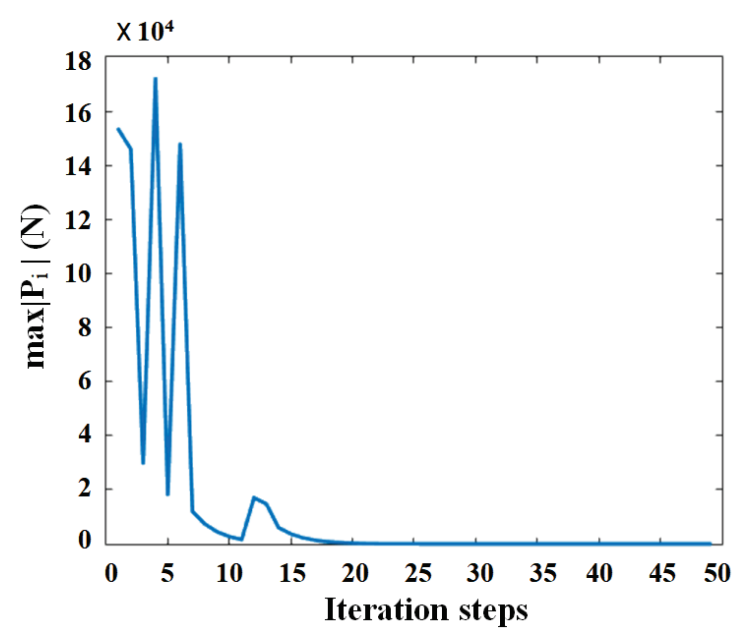

Fig.15 History of Step-by-Step UFI of ellipsoidal Levy form cable dome

\section{Example VI: Asymmetric Levy form cable dome}

The sixth example is a Levy form cable dome, as shown in Fig. 16, with circular boundary but asymmetric geometry. The dome has three rings of hoops and an inner ring. The diameter of boundary is $100 \mathrm{~m}$, and the diameter of three hoops are $75 \mathrm{~m}, 50 \mathrm{~m}$ and $20 \mathrm{~m}$, respectively. Each hoop is divided into 12 parts. There are 252 cables and 48 struts. The number of free nodes is 48 , i.e., the number of variable coordinates is $48 \times 3=144$.

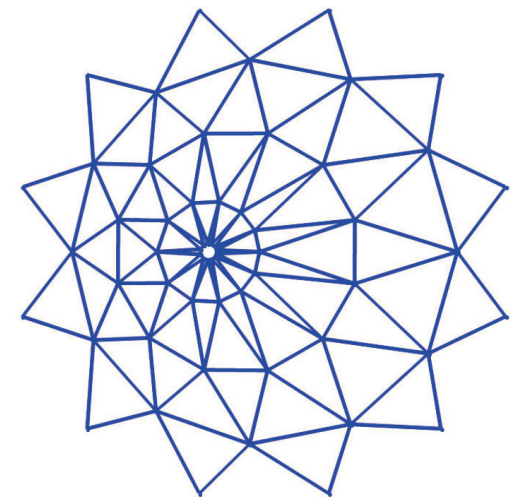

(a) top view

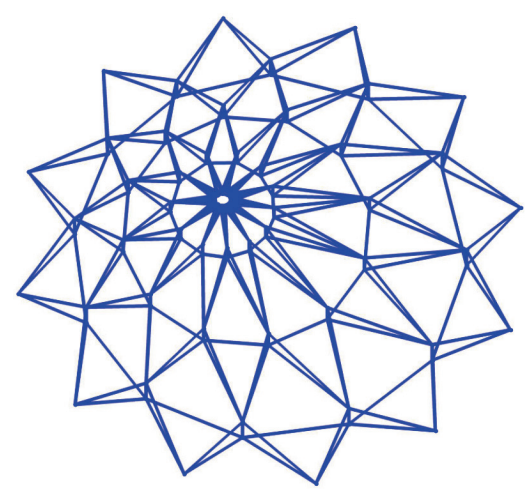

(b) perspective view

Fig.16 Asymmetric Levy form cable dome

The history of UFI of initial geometry is shown in Fig. 17. The initial prestress values of cables and struts are $200000 \mathrm{~N}$ and $-50000 \mathrm{~N}$, respectively. The unbalanced force varies around 
$1.36 \times 10^{4} \mathrm{~N}$ after the 100 th iteration, which verifies the infeasibility of initial geometry. Therefore, the Step-by-Step UFI is carried out with $N_{1}$ and $N_{2}$ equal to $5000 \mathrm{~N}$ and $-5000 \mathrm{~N}$, respectively. The process converged at the 44th iteration with the final unbalanced force $0.19 \mathrm{~N}$. The history of iteration result is shown in Fig. 18.

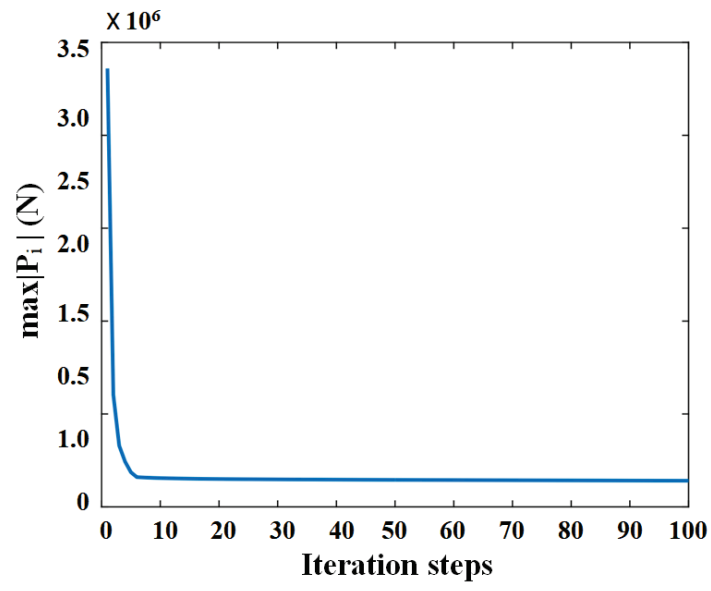

Fig.17 History of UFI of initial geometry of asymmetric Levy form cable dome

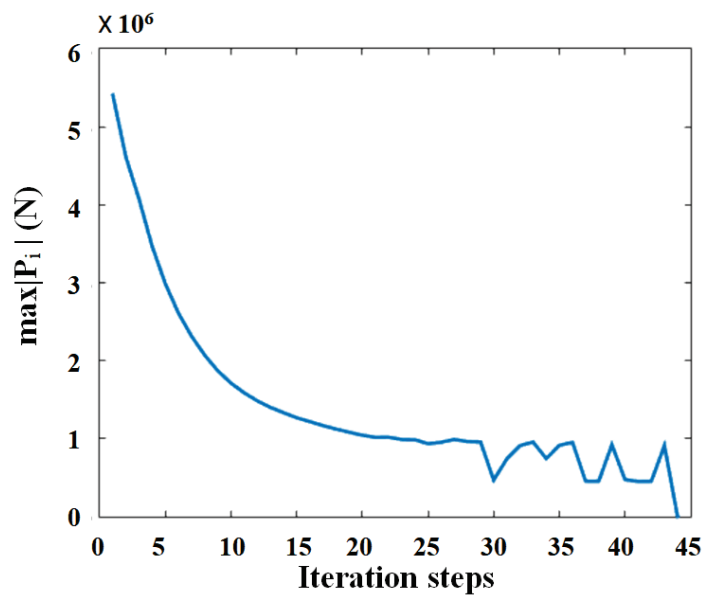

Fig.18 History of Step-by-Step UFI of asymmetric Levy form cable dome

The initial and final geometries are compared in Fig. 19. In the horizontal direction, the two models almost coincide. The average nodal distance between initial and final geometry is $0.45 \mathrm{~m}$, and the maximum distance is $1.13 \mathrm{~m}$, which exists at the lower inner ring. Note that the 
difference in the hoops are sufficiently small compared with the size of the dome.

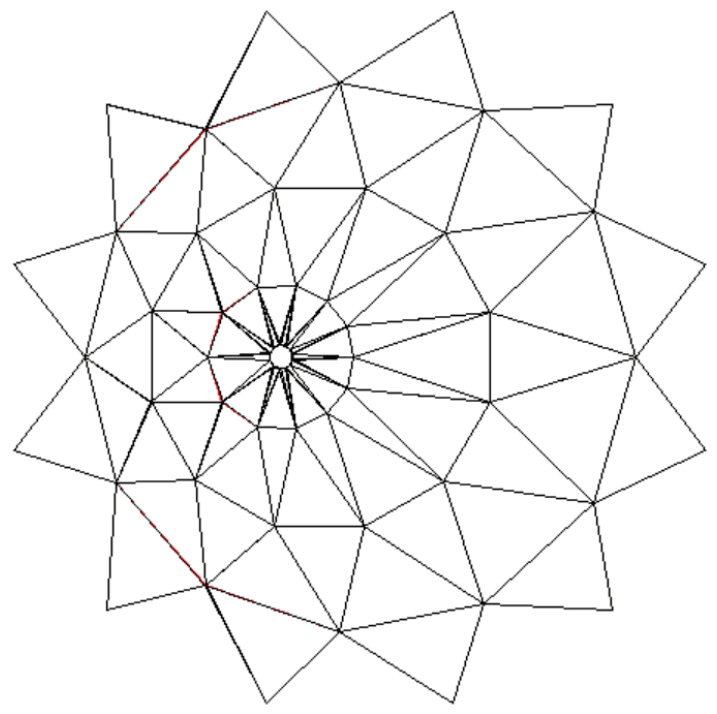

(a) horizontal view

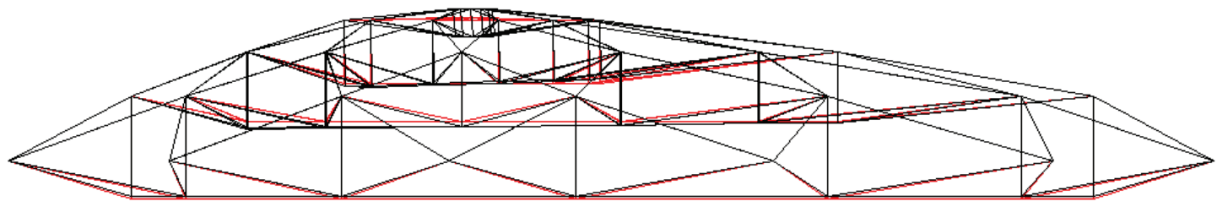

(b) vertical view

Fig.19 Comparison of initial and final geometries (red: initial; black: final)

\section{Conclusion}

Methods of prestress design and form finding of cable-strut structures have been presented. The results obtained in this paper are summarized as follows:

1. Feasibility of geometry is defined so that the prestresses of cables and struts in the selfstress mode are in tension and compression, respectively. A simple iterative process called Unbalanced Force Iteration (UFI) method has been proposed to judge feasibility of a geometry and find the self-stress modes of a cable dome structure.

2. Self-stress modes can be directly obtained easily through UFI method, if initial geometry is feasible. Furthermore, the number of static indeterminacy, which is equal to the number of 
self-stress modes, can be obtained using the process of UFI without resort to the computationally expensive SVD.

3. For structures with infeasible geometry, feasible nodal coordinates can be found by Stepby-Step UFI that modifies the nodal locations by the displacements due to the unbalanced forces. It has been shown that feasibility of self-stress mode can be achieved through small modification of geometry.

4. New configurations with non-circular shape or asymmetric geometry presented in this paper gives alternative choices for design of cable domes with a variety of architectural assignment.

\section{Acknowledgement}

This work is sponsored by the National Natural Science Foundation of China (Grant No. 51478310) and the Science and Technology Program of Ministry of Housing and Urban-Rural Development of China (No. 2016-K5-062). The authors also appreciate the support provided by the Chinese Scholarship Council (File No. 201706250070) that enables the author to conduct research with Prof. M. Ohsaki at Kyoto University.

\section{Reference}

[1] Snelson, K. "Snelson on the Tensegrity Invention." International Journal of Space Structures 11.1-2 (1996): 43-48.

[2] Fuller, R. Buckminster, and E. J Applewhite. Synergetics: Explorations in the Geometry of Thinking. Macmillan Pub. Co, 1975.

[3] Zhang, J. Y., and M. Ohsaki. "Adaptive force density method for form-finding problem of tensegrity structures." International Journal of Solids and Structures 43.18-19 (2006): 5658-5673. 
[4] Zhang, J. Y., and Ohsaki, M. “Tensegrity Structures: Form, Stability and Symmetry.” Springer (2015).

[5] Geiger, David H., Andrew Stefaniuk, and David Chen. "The design and construction of two cable domes for the Korean Olympics." Proc. of the IASS Symposium on Shells, Membranes and Space Frames. 1986.

[6] Levy, Matthys P. "The Georgia Dome and beyond: achieving lightweight-longspan structures." Spatial, Lattice and Tension Structures. ASCE, 1994.

[7] Robison, Rita. "Fabric meets cable." Civil Engineering 59.2 (1989): 56.

[8] Geiger, D. H. "Design details of an elliptical cable dome and a large span cable dome (210 m) under construction in the United States." Proc. of the IASS ASCE International Symposium on Innovative Applications of Shells and Spatial Forms. 1988.

[9] Pellegrino, Sergio. "A class of tensegrity domes." International Journal of Space Structures 7.2 (1992): 127-142.

[10]Lazzari, Massimiliano, et al. "Dynamic behavior of a tensegrity system subjected to follower wind loading." Computers \& structures 81.22-23 (2003): 2199-2217.

[11] Gossen, P., D. Chen, and E. Mikhlin. "The First Rigidly Clad" Tensegrity" Type Dome, The Crown Coliseum, Fayetteville, North Carolina." Spatial Structures in New and Renovation Projects of Buildings and Construction: theory, investigations, design, erection (1998): $477-484$.

[12]Lee, Kyoungsoo, SangEul Han, and Taehyo Park. "Stabilization process analysis of cable dome structure." International Journal of Steel Structures 12.4 (2012): 495-507. 
[13] Ma Q., Chen Z., Yan X.. Analysis of new type of cable dome with rigidly cladding in arena of Tianjin University of Technology. Proc. of the IASS on Spatial Structures in the 21st Century. 2016.

[14] Yuan, X., Chen L., Dong S.. "Prestress design of cable domes with new forms." International Journal of Solids and Structures 44.9 (2007): 2773-2782.

[15]Guo, J., Zhu M.. "Negative Gaussian curvature cable dome and its feasible prestress design." Journal of Aerospace Engineering 29.3 (2015): 04015077.

[16]Uzun, F.. "Form-finding and analysis of an alternative tensegrity dome configuration." Advances in Structural Engineering 20.11 (2017): 1644-1657.

[17] Quagliaroli, M., et al. "The role of prestress and its optimization in cable domes design." Computers \& Structures 161 (2015): 17-30.

[18]Lu, J., et al. "Form-finding analysis for a new type of cable-strut tensile structures generated by semi-regular tensegrity." Advances in Structural Engineering 20.5 (2017): 772-783.

[19]Pellegrino, Sergio. "Structural computations with the singular value decomposition of the equilibrium matrix." International Journal of Solids and Structures 30.21 (1993): 30253035 .

[20] Yuan, X. F., and S. L. Dong. "Integral feasible prestress of cable domes." Computers \& structures 81.21 (2003): 2111-2119.

[21]Zhang, J. Y., M. Ohsaki, and Y. Kanno. "A direct approach to design of geometry and forces of tensegrity systems." International Journal of Solids and Structures 43.7-8 (2006): 22602278.. 
[22] Wang Z, Yuan X, Dong S. "Simple approach for force finding analysis of circular Geiger domes with consideration of self-weight." Journal of Constructional Steel Research 66.2 (2010): 317-322.

[23] Tran H C, Park H S, Lee J. "A unique feasible mode of prestress design for cable domes." Finite Elements in Analysis and Design 59 (2012): 44-54.

[24]Guo, J. and J. Jiang. "An algorithm for calculating the feasible pre-stress of cable-struts structure." Engineering Structures 118 (2016): 228-239.

[25] Ye, J., et al.. "Simulation of construction shape-forming process of cable domes." Science China Technological Sciences 55.1 (2011): 101-116.

[26]Barnes, Michael R. "Form finding and analysis of tension structures by dynamic relaxation." International journal of space structures 14.2 (1999): 89-104.

[27] Schek, H. J.. "The force density method for form finding and computation of general networks." Computer Methods in Applied Mechanics and Engineering 3.1 (1974): 115-134.

[28]Tibert, A. G., and Sergio Pellegrino. "Review of form-finding methods for tensegrity structures." International Journal of Space Structures 26.3 (2011): 241-255.

[29] Lu, J., et al. "Form-finding analysis of irregular tensegrity structures by matrix iteration." Advanced Steel Construction 11.4 (2015): 507-516.

[30]Lee, S., et al. (2016). "A fully automatic group selection for form-finding process of truncated tetrahedral tensegrity structures via a double-loop genetic algorithm." Composites Part B: Engineering 106 (2016): 308-315.

[31] Yuan, X.-F., et al. (2017). "Form-finding of tensegrity structures based on the Levenberg- 
Marquardt method." Computers \& Structures 192 (2017): 171-180.

[32] Bletzinger K U, Ramm E. "A general finite element approach to the form finding of tensile structures by the updated reference strategy." International Journal of Space Structures 14.2 (1999): 131-145.

[33] Pagitz, M., and JM Mirats Tur. "Finite element based form-finding algorithm for tensegrity structures." International Journal of Solids and Structures 46.17 (2009): 3235-3240.

[34]Klinka K K, Arcaro V F, Gasparini D. "Form finding of tensegrity structures using finite elements and mathematical programming." J Mech Mater Struct 7.10 (2012): 899-907.

[35]Horn, Roger A., and Charles R. Johnson. Matrix analysis. Cambridge university press, 1990.

[36] Higham, Desmond J., and Nicholas J. Higham. MATLAB guide. Vol. 150. Siam, 2016.

[37] Canonsburg, P. A. "ANSYS Structural Analysis Guide, Release 18.0." SAS. IP, Inc 17 (2017).. 